\title{
Calls for Interaction: The More the Better? User Experience of 3D Carousel and Additional Interaction Techniques
}

\author{
S. Shyam Sundar ${ }^{1,2}$, Saraswathi Bellur ${ }^{1}$, Jeeyun $\mathrm{Oh}^{1}$, and Haiyan Jia ${ }^{1}$ \\ ${ }^{1}$ Media Effects Research Laboratory, \\ The Pennsylvania State University, USA \\ ${ }^{2}$ Department of Interaction Science, \\ Sungkyunkwan University, Seoul, South Korea \\ \{sss12, saras, jzo120, hjia\} @psu.edu
}

\begin{abstract}
We perform a user study to investigate the psychological consequences of adding interaction techniques to the interface. In a betweensubjects experiment $(\mathrm{N}=143)$, we explore how (i) variations in sheer number of interaction techniques and (ii) addition of a novel technique, i.e., 3D carousel, influence the volume of users' actions, their memory, perceptions of interactivity, as well as their attitudes and behaviors toward a website. Power usage is examined as a potential moderator. First-cut findings from self-reports and $\log$ data indicate that the 3D carousel feature has a strong impact on user experience, both positive and negative. It also moderates the curvilinear effect of adding traditional interaction techniques to the interface.
\end{abstract}

Keywords: Interaction Techniques, 3D carousel, user experience, user engagement.

\section{Introduction}

The range and richness of interaction techniques on websites offer users unprecedented variety and choice in interacting with an interface. For instance, the 3D carousel is fast becoming a common feature on websites, joining a growing cadre of interaction tools. However, little is known about the user experience created by these techniques. Does the increased number of calls for interaction necessarily enhance user experience? How does this feature combine with other techniques on the interface to influence higher-order outcomes? With this in mind, the purpose of our study is twofold: (1) to examine the effect of the addition of interaction techniques to user experience, their memory, attitudes and behaviors, and (2) to specifically explore the effect of the $3 D$ carousel technique, and how it affects user experience?

\section{Effects of Interaction Techniques}

We define interaction techniques as any feature embedded in the website that offers various modes of interactions to users, based on the tripartite model of interactivity effects proposed by Sundar [1], which distinguishes between modality interactivity, 
source interactivity (customization) and message interactivity (contingent exchanges of messages). Interaction techniques come under the first of the three kinds of interactivity, in that they afford various modes of accessing information on an interface. Different types of interaction techniques add to the interactivity potential of the interface. However, literature suggests that the effects of interactivity are not always linear or positive. Empirical observations have revealed a so-called "interactivity paradox" [2], with studies pointing to an optimal threshold beyond which the effects of interactivity diminish [3]. Just what, how or when exactly is such a threshold reached remains an open question that is worthy of investigation. Aside from increases in the availability of interaction techniques, the presence of novel techniques such as the 3D carousel, with its characteristically revolving motion, invite active user action (e.g., flipping or swiping) that add to its interactivity potential. Its constantly rotating feature makes the $3 \mathrm{D}$ carousel playful, engaging and capable of providing rich forms of interaction, as claimed by Björkskog et al [4]. However, such enthusiasm for it is yet to be validated empirically with users, especially when it coexists with other interaction techniques on the interface. Therefore, we experimentally examine how the addition of 3D carousel, combined with other interaction techniques, affects user experience and the degree of user engagement with a Web interface.

\section{Method}

We conducted a 4 (Click only, Click + Slider, Click + Slider + Drag, Click + Slider + Drag + Mouseover) $\times 2$ (presence or absence of 3D carousel) between-subjects fullycrossed factorial experiment. Participants recruited from undergraduate classes $(N=$ 143; 93 females) were randomly assigned to one of the eight conditions. Eight prototype websites were especially constructed for this experiment based on an online exhibition entitled "Guitar/Bass Timeline" [5]. Except for the number of interaction techniques employed, all eight versions of the prototype website shared the same content and page layout. We embedded the manipulation of 3D carousel in the homepage (Figure 1). Whenever participants clicked on a guitar in the homepage, the site played a brief audio-clip of a classic riff produced by that particular guitar/bass.
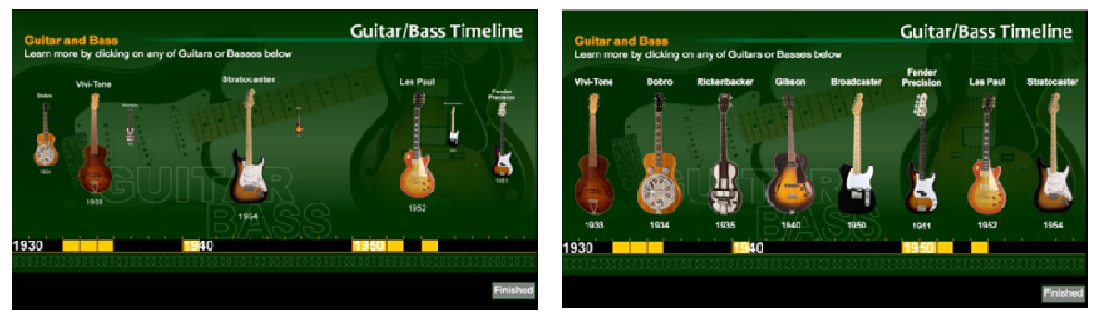

Fig. 1. Homepage with 3D carousel vs. without 3D carousel

In both conditions, clicking on one of the guitars took participants to the next layer of site content, which featured a slider (for going across a timeline), a drag feature (allowing users to drag a guitar-pick on the instrument for more information), and/or a mouseover function (for accessing embedded textual information in so-called "hotspots"). Examples of stimuli are accessible at <http://tinyurl.com/3f8ypmk $>$. The 
websites for all eight conditions were elaborately pretested for usability. Based on literature which identifies browsing as the quintessential interaction task [6], we instructed our study participants to browse the stimulus website fully and completely. To make the task as realistic as possible, no time constraints were imposed, nor were the participants told that their memory for site's content would be tested later in the study. All questions were measured on a 7-point Likert scale, except for recognition and recall memory measured through multiple-choice and open-ended questions. Power usage was measured via 12 items about the degree of participants' liking of technology and their extent of use [7] $(\alpha=.83)$. Perceived interactivity was measured by 3 items (for e.g., the degree to which it allows them to perform lots of actions) ( $\alpha=$ .76) based on [8]. Perceived ease of use was measured by 3 items $(\alpha=.74)$ adapted from [9]. Behavioral intention toward website was measured by 6 questions $(\alpha=.93)$ [10]. We collected log data using jQuery to measure user actions (average frequency of page visits and average number of user interactions per hotspot).

\section{Results}

An interaction effect showed that the addition of interaction techniques to the interface served to enhance user perceptions of interactivity only in the absence of 3D carousel, $F(3,134)=4.11, p<.01$. The relationship between the number of interaction techniques and perceived ease of use assumed an inverted-U shape when the $3 \mathrm{D}$ carousel feature was present on the interface, $F(3,134)=4.39, p<.01$ (see Figure 2). The analysis of behavioral intentions (e.g., forwarding and recommending the site to others) yielded a similar inverted-U interaction, $F(3,134)=3.01, p<.05$ (Figure 3 ).

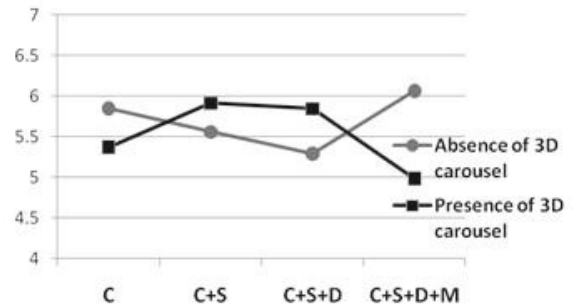

Fig. 2. Perceived ease of use

(C: Click, S: Slide, D: Drag, M: Mouseover)

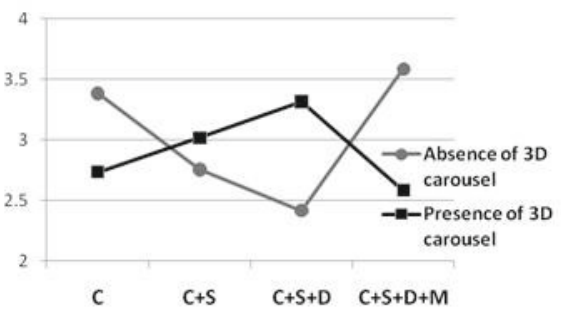

Fig. 3. Behavioral intentions (C: Click, S: Slide, D: Drag, M: Mouseover)

A significant main effect revealed that $3 \mathrm{D}$ carousel served to degrade aural recognition memory of the guitar riffs, $F(1,134)=6.17, p<.05$. In terms of user actions, users were most likely to interact with information hotspots when 3D carousel was present, $F(1,131)=11.62, p<.001$. However, users tended to visit the homepage (i.e., the main page with the guitars) less frequently when $3 \mathrm{D}$ carousel was present, $F(1,132)=3.98, p<.05$. Power usage positively predicted perceptions of interactivity, $F(1,134)=8.14, p<.01$, and behavioral intentions toward the site, $F$ $(1,134)=3.95, p<.05$. As a moderator, power usage was positively associated with recall memory for content in the $3^{\text {rd }}$ and $4^{\text {th }}$ conditions, but inversely related to recall when only two basic interaction techniques were present, $F(3,135)=3.68, p<.05$. 


\section{Discussion}

Taken together, the 3D carousel boosted perceptions of interactivity, so much so that it overrode common interaction techniques such as click, slider, and drag. It also stimulated user interaction. However, this appeal of 3D carousel came at a cognitive cost, degrading users' memory for audio that accompanied the guitars and inhibiting their tendency to visit the homepage. It negatively affected perceived ease of use and behavioral intentions when the study website was saturated with four interaction techniques, even when users reported that a site with 3D carousel was easier to use and were likely to recommend it. This parallels previous findings [11] that the 3D carousel is more suitable for aesthetic appeal than task efficiency. In addition, more interaction techniques aided the information uptake for power users, encouraging us to consider individual differences relating to technology competency when predicting user experience with new as well as multiple combinations of interaction techniques.

Acknowledgments. This research is supported by the U.S. National Science Foundation under Grant No. IIS-0916944 and the Korea Science and Engineering Foundation under World Class University (WCU) Grant No. R31-2008-000-10062-0.

\section{References}

1. Sundar, S.S.: Social psychology of interactivity in human-Website interaction. In: Joinson, A.N., McKenna, K.Y.A., Postmes, T., Reips, U.-D. (eds.) The Oxford Handbook of Internet Psychology, pp. 89-104. Oxford University Press, Oxford (2007)

2. Bucy, E.P.: The debate. The Information Society 20(5), 371 (2004)

3. Sundar, S.S., Kalyanaraman, S., Brown, J.: Explicating web site interactivity: Impression formation effects in political campaign sites. Communication Research 30, 30-59 (2003)

4. Björkskog, C., Jacucci, G., Lorentin, B., Gamberini, L.: Mobile implementation of a web 3D carousel with touch input. In: Proc. of the 11th International Conference on HumanComputer Interaction with Mobile Devices and Services, pp. 1-4. ACM, New York (2009)

5. http://www. empsfm.org/flash/guitbass/index.html

6. Zhai, S., Smith, B.A., Selker, T.: Improving Browsing Performance: A study of four input devices for scrolling and pointing tasks. In: Proc. of the 6th IFIP Conference on HumanComputer Interaction, pp. 286-292 (1997)

7. Marathe, S.S., Sundar, S.S., Bijvank, M.N., van Vugt, H., Veldhuis, J.: Who are these power users anyway? Building a psychological profile. Paper presented at the 57th Annual Conference of the International Communication Association (2007)

8. Kalyanaraman, S., Sundar, S.S.: The psychological appeal of personalized online content in Web portals: Does customization affect attitudes and behavior? Journal of Communication 56, 110-132 (2006)

9. Davis, F.D.: Perceived usefulness, perceived ease of use, and user acceptance of information technology. MIS Quarterly 13(3), 319-339 (1989)

10. Hu, Y., Sundar, S.S.: Effects of online health sources on credibility and behavioral intentions. Communication Research 37, 105-132 (2010)

11. Wang, S., Poturalski, M., Vronay, D.: Designing a generalized 3D carousel view. In: CHI 2005 Extended Abstracts on Human Factors in Computing Systems, pp. 2017-2020. ACM Press, New York (2005) 